\title{
Case Report: Students' Perception on Government Policy of Routine HIV Testing: A Case of University of Zambia Ridgeway Campus
}

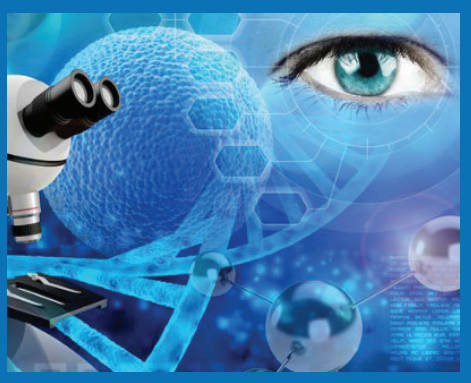

Author Name:

Chibochi Kaminsa, Chirwa Simon,

Kalembwe Vincent, Mukomenji

Celestine, Martha Mbewe Mwelwa,

Natalia Mbewe Shitima, Susan

Mutemwa, Caroline Zulu

University of Zambia, School of Nursing Sciences

Corresponding Author:

Caroline Zulu

Citation: Caroline Zulu,

Students' Perception on Government

Policy of Routine HIV Testing: A Case of University of Zambia Ridgeway

Campus

\section{Received Date: 15th June 2021}

Published Date: 07 September 2021

\section{Copyrights: Caroline Zulu}

This is an open access article distributed under the Creative Commons Attribution License, which permits unrestricted use, distribution, and reproduction in any medium, provided the original work is properly cited.

\begin{abstract}
Background

HIV Routine testing to all hospital attendants offers testing to all patients irrespective of their presenting illness even if it has nothing to do with HIV. This approach differs from voluntary counselling and testing, which is client-initiated. Adolescents and young adults especially those in colleges are a sexually active group of people which makes them prone to HIV infection. Therefore, there was need to conduct this study to determine the perception of students on routine HIV testing at the University of Zambia (Ridgeway campus) to determine their attitude towards HIV testing.
\end{abstract}

\section{Design and Methods}

A cross sectional quantitative study was conducted at UNZARidgeway hostels using stratified random sampling to select study participants which included all males and females who were on campus. A semi-structured interview questionnaire was applied to collect data which was analysed using SPSS version 23software. A Chi-square test was applied for descriptive analysis and results were presented in tables and charts.

\section{Results}

Majority indicated that routine HIV testing was a good policy and supported it; though surprisingly $94 \%$ of the students were knowledgeable about routine HIV testing and yet their knowledge did not affect their perception of the policy. However, their experiences, expectations and beliefs affected students' perception on routine HIV testing.

\section{Conclusion}

Health practitioners should have positive attitude and good work culture in order to uphold students' positive perception so as to encourage a culture of HIV testing and is free from stigma.

Key words:

Perception, HIV testing, students

\section{Introduction}

In 2017, there was about 36.8 million people living with HIV globally and about 48149 cases were reported as newly infected. The prevalence among people aged 15 to 49 was $11.3 \%$ (WHO, 2019). WHO estimates that only about 10 per cent of persons living with HIV in low and middle-income countries know their HIV status (WHO/UNAIDS, 2007). Over 95\% of the global total of all AIDS cases are in the developing world. Overwhelming majority of all infections globally are acquired through unprotected sexual intercourse, with at least $70 \%$ resulting from heterosexual intercourse (PEPFAR, 2017).In 2008, about $68 \%$ of people living with HIV were in sub-Saharan Africa with around 35\% in eight countries alone. In 2009, the (Great eight countries) met in Scotland and committed to achieving universal access to HIV prevention, care and treatment by 2010. (WHO, 2017). The UNAIDS 90-90-90 targets, set for 2020, are that 90\% of HIV-positive individuals know their HIV-positive status, $90 \%$ of those who know their HIV-positive status are on antiretroviral therapy (ART), and $90 \%$ of those taking ART are virally suppressed. These targets correspond to $81 \%$ of HIV-positive individuals being 
on ART and $73 \%$ of HIV-positive individuals being virally suppressed, with the overall goal of improving the health of people living with HIV and decreasing HIV transmission at the population level. (UNAIDS, 2014).

There has been widespread concern about the slow uptake of Voluntary Counselling and Testing (VCT) in many parts of sub-Saharan Africa (Wanyenze, Nawavvu et al. 2008). VCT is a cornerstone of cost-effective HIV prevention and linkage to expanding HIV treatment in low-resource settings (Weiser, Heisler et al.2006). However, some of the most significant barriers to HIV testing identified in sub-Saharan Africa include lack of access to VCT, lack of access to antiretroviral (ARV) therapy, and HIV-related stigma (Chandisarewa, Stranix-Chibanda et al. 2007) proved to be a challenge, hence, the introduction of the routine HIV testing. (Onyeonoro, 2014). In 2004, the united nations HIV/ AIDS (UNAIDS) decided on changing the way of testing and treating for HIV so as to sharply increase the number of people being tested for HIV by introducing the routine HIV testing, counselling and treatment (HIV/AIDS and Scholar), a departure from the traditional voluntary counselling and testing (VCT) model as VCT involves people self-presenting for testing at their local medical facilities if they believe they have been exposed to HIV which led to poor uptake of VCT, despite decades of AIDS education campaigns, it is reflected and estimates that the vast majority had no knowledge of their HIV status(Rennie and Behets 2006).

In the meantime, Zambian was still sticking to the voluntary HIV counselling and testingadoptedin March, 1999, as a way for individuals who are not affected to protect themselves from getting infected with HIV and those infected can live a healthy lifestyle, during which, ART was only given when the CD4 count was below 200 copies per litter (Ministry of health, 2004). This helped to reduce the spread of HIV but there was reduced rates of voluntary testing by the community due to HIV related stigma, which led to slow rate of combating the HIV epidemic. (MoH, 2010).By 2016, Zambia had 59000 (52 000 - 69000$)$ new HIV infections and 21000 (17 000 - 28 000) AIDS-related deaths. With these rates of new infections, the government of Zambia adopted the routine HIV testing in order to fast track the HIV epidemic control through testing and counselling which could lead to more individuals being tested. This policy was made effective in September, 2017 by the ministry of health. This strategy is currently being used by the health sector with the aim of immediately testing clients regardless of the CD4 count started on treatment if the person is found to be HIV positive and of improved linkage to care to all infected individuals. Objectives of this study were to determine students' perceptions on routine HIV testing by assessing students 'knowledge level,ascertainingtheirbeliefs, experiences and expectations on routine HIV testing.

\section{Design and Methods}

A quantitative cross-sectional descriptive approach was used in this study to quantify the problem by way of generating numerical data or data that can be transformed into usable statistics. Therefore, in this study it was used to quantify knowledge, beliefs, experiences and perception of $n=186$ university students using stratified random sampling towards routine HIV testing conducted at the University of Zambia (ridgeway campus). Written structured questionnaires were administered using oneto-one interviews in order to collect data.

\section{Results}

Table 1: Social-demographics

\begin{tabular}{|l|l|l|}
\hline Variables & Categories & $\begin{array}{l}\text { Frequency } \\
\text { (Percentage) }\end{array}$ \\
\hline \multirow{2}{*}{ Sex } & Females & $58(31 \%)$ \\
\cline { 2 - 3 } & Males & $128(69 \%)$ \\
\hline \multirow{2}{*}{ Total } & Female + male & $186(100 \%)$ \\
\hline \multirow{2}{*}{ Total } & 16 to 30 years & $166(89 \%)$ \\
\cline { 2 - 3 } & 31 and above & $20(11 \%)$ \\
\hline \multirow{2}{*}{ Marital status } & $16-30+>30$ & $186(100 \%)$ \\
\hline Total & Single & $168(90 \%)$ \\
\cline { 2 - 3 } Religion & Married & $18(10 \%)$ \\
\hline Total & Single + married & $186(100 \%)$ \\
\cline { 2 - 3 } & Christian & $165(89)$ \\
\cline { 2 - 3 } & Others & $21(11 \%)$ \\
\hline
\end{tabular}

Table 1 above shows 58 (31\%) of participants were females and $128(69 \%)$ were males. $166(89 \%)$ were aged between 16 to 30 years and $20(11 \%)$ aged 30 and above. As expected more, 168 (90\%) were single whilst $18(10 \%)$ were married and $165(89 \%)$ of the respondents were Christians and 21 (11\%) belonged to other religions.

\section{Figure 1. Respondents per programme}

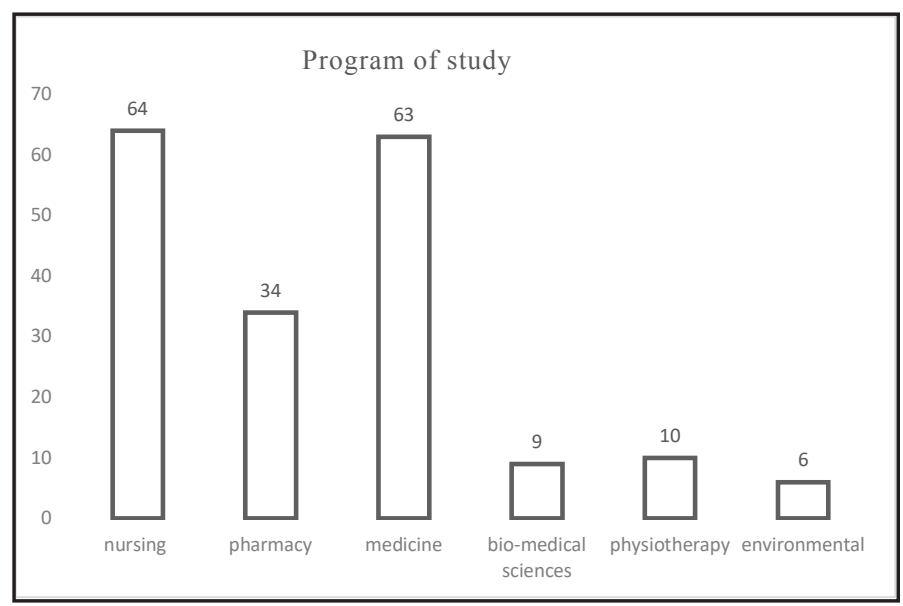

Figure 2 shows the students per programme of study 64 (34.40\%) were nursing, 34 (18.28\%), pharmacy,63 (33.87\%) medical,9biomedical sciences physiotherapy $10(4.84 \%)$ and 6 (3.23\%) environmental science students.

\section{Figure 2.Acceptabilityof Routine HIV Testing}

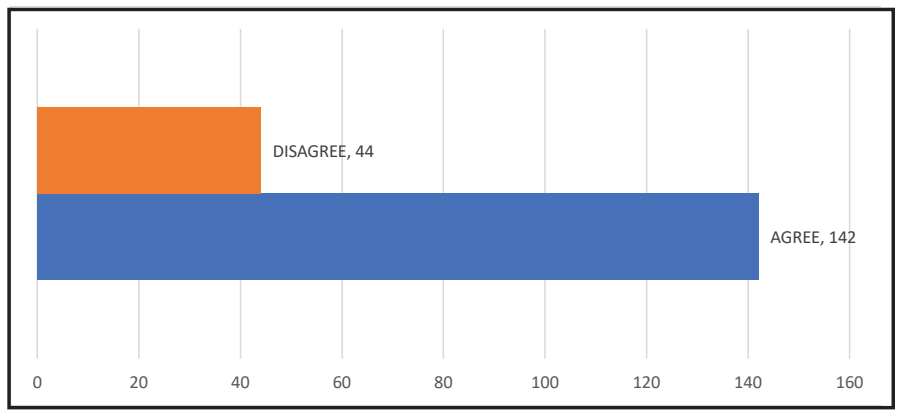


Majority of the respondents were in support of the routine HIV testing. Figure 2 above shows that $142(76 \%)$ of the respondents agreed that it was right to be tested for HIV every time they visit the health centre for any health problem, while $44(26 \%)$ disagreed to this.

Table 2: Opinion over routine HIV testing policy

\begin{tabular}{|c|c|c|}
\hline Opinion & Frequency & Percentage \\
\hline Good Policy & 151 & $81 \%$ \\
\hline Bad policy & 35 & $19 \%$ \\
\hline Total & 186 & $100 \%$ \\
\hline
\end{tabular}

The table above shows that 151 (81\%)of respondents expressed that the routine HIV testing policy was a good policy while $35(19 \%)$ thought it was a bad policy.

Results of knowledge of the respondents on routine HIV testing showed that $94 \%$ understood it as an HIV test done every time someone visits the health centre as part of the screening process and only $6 \%$ thought that it was the same as voluntary HIV testing.

\section{Table 3: Reason for unwillingness to get tested for HIV}

\begin{tabular}{|l|l|l|}
\hline & Frequency & Percentage \\
\hline $\begin{array}{l}\text { Fear of knowing } \\
\text { their results }\end{array}$ & 182 & $98 \%$ \\
\hline Time consuming & 04 & $2 \%$ \\
\hline Total & 186 & $100 \%$ \\
\hline
\end{tabular}

Table 3 above on unwillingness of students to go to the hospital and be tested for HIV even if they thought that they had an illness that is not related to HIV was due to fear of a positive result $(98 \%)$ of while only $2 \%$ indicated that it was because the process of testing was time consuming.

\section{Figure 3: Tested for HIV on their last clinic visit}

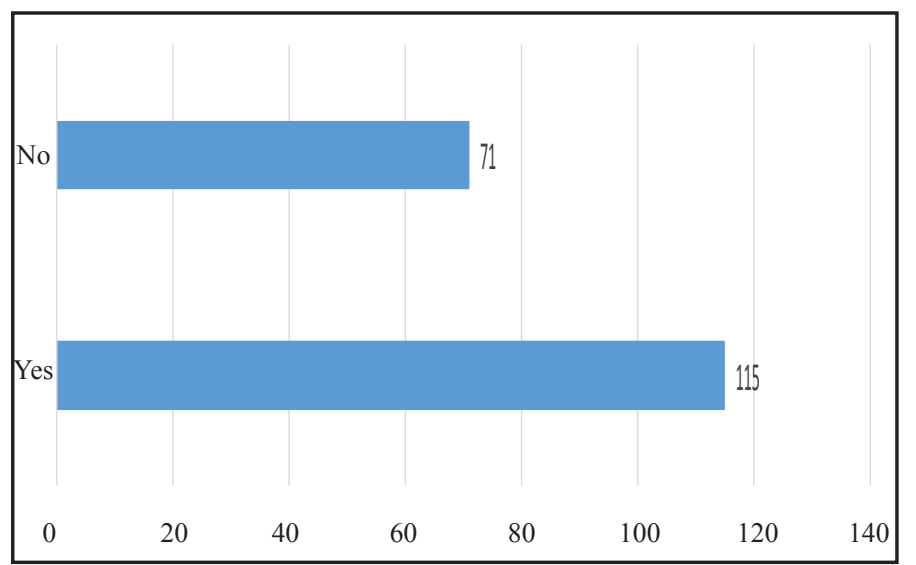

Experiences and expectations as shown figure 3 above indicate that $71(47 \%)$ of the respondents did not get tested for HIV the last time they visited a health centre to seek for medical attention while $115(53 \%)$ of the respondents did get tested.
Table 4: Students opinion on policy vs HIV test done

\begin{tabular}{|c|c|c|c|c|c|}
\hline \multicolumn{6}{|c|}{ Students opinion on policy vs HIV test done } \\
\hline & & \multicolumn{2}{|c|}{ Was the test done } & \multirow{2}{*}{ Total } & \multirow{2}{*}{ P-Value } \\
\hline & & Yes & No & & \\
\hline \multirow{2}{*}{$\begin{array}{l}\text { Opinion } \\
\text { on the } \\
\text { routine } \\
\text { HIV } \\
\text { policy }\end{array}$} & $\begin{array}{l}\text { Good } \\
\text { policy }\end{array}$ & 89 & 62 & 151 & \multirow{3}{*}{0.001} \\
\hline & $\begin{array}{l}\text { Bad } \\
\text { policy }\end{array}$ & 10 & 25 & 35 & \\
\hline \multicolumn{2}{|l|}{ Total } & 99 & 87 & 186 & \\
\hline
\end{tabular}

The table above shows that there is an association between students' opinion over the routine HIV testing policy and being tested for HIV at a p-value of 0.001 , (81\% of respondents) showing a positive support that the policy was good. It was also observed that of the 115 respondents that said they had been tested, $60 \%$ underwent pre-counselling, intra and post counselling, while $40 \%$ were tested without being counselled.

\section{Discussion}

Key findings indicate that there was overwhelming agreement and support $(81 \%)$ by students on the routine policy for HIV testing, saying that it was a good policy. However, students' experiences had an effect on their perception of routine HIV testing. Surprisingly though most of them were knowledgeable, their knowledge levels, expectations and beliefs had no significant influence on their perception of routine HIV testing. Majority of student's (94\%) understood that routine HIV testing is done every time someone visitsa health care facility as part of the screening process. It was therefore not surprising that upon applying Pearson's Chi-square test for cross tabulation between knowledge and students' opinion on routine HIV testing it confirmed that the understanding of students on routine HIV testing affected their opinion over the routine HIV testing. These study findings are in agreement with the findings in the study conducted in KwaZulu-Natal province, South Africa, which revealed that the majority of the respondents' demonstrated knowledge of HIV testing and were in support the policy(Gumede, S. D., \& Sibiya, M. N. 2018).

Expectations and experiences shape our thoughts and how we perceive things and the world around us, and it also affects how we respond to certain things and activities (Nally A. et all2019). In this study, $53 \%(n=115)$ were tested for HIV as part of the screening process from which; $60 \%$ had pleasant experiences as they underwent pre-counselling, intra-counselling and post counselling, while $40 \%$ were tested without being counselled. This could explain the overwhelming agreement and support by students of the routine HIV testing policy despite the fact that the target group was entirely among health students educated in HIV disease and so have also learnt the benefits of early diagnosis through routine HIV testing which facilitates diagnosing of HIV infection and so quicken the initiation of treatment.

As researchers we may assume that beliefs will affect the routine HIV testing, but this study scientifically proves this assumption wrong between beliefs and the perception of students towards routine HIV testing which gave a P-value of 0.574 , showing no relationship between the beliefs and the perception of students towards routine HIV testing at UNZA(Ridgway campus). Similar findings were discovered in a study conducted, on factors affecting HIV testing among youths in Tanzania that beliefs such as stigma and fear of being found HIV positive did not affect 
the perception of youths towards HIV testing (Manongi, R., et al. 2014).

Similar observations were made when a theory-based research was conducted in some universities in southern African countries addressing factors influencing HIV testing among college students in which they explored the routine HIV testing via a conceptual framework that integrates the health belief model with emotion and communication factors. Results showed that students' low emotional self-efficacy was a barrier for adopting routine HIV testing, they were however open to receiving cues to action via confidential HIV-testing related communication from health professionals. The study concluded that normalizing HIV testing as a primary care routine for harm avoidance or reduction of HIV infections would increase perceived benefits of testing (CDC, 2012). Findings of both researches can significantly contribute to combat the HIV epidemic by having more people testing and so accessing care and treatment.

\section{Conclusion}

HIV/AIDS continues to be a worldwide burden especially in developing countries like Zambia.The policy of routine HIV testing seems well understood by the students and if this age group positively responded by undertaking it, this can help to lower the burden of HIV disease which it impacts on health and productivity of young people and the country at large. Majority of UNZA (Ridgeway campus) students had a positive perception on routine HIV testing policy as they expressed that the introduction of this policy was good and many were in support of its implementation that is supporting the government's move on introducing the implementation of routine HIV testing policy. However, results show that experiences affect the perception of students towards routine HIV testing therefore, the health practitioners should have positive attitudes and good work cultures in order to help students have good experience at the health facilities.

\section{References}

Broeckaert. L., Challacombe. L, (2017), the routine offers of HIV testing in primary care settings.

Centers for Disease Control and Prevention (CDC). Estimated HIV incidence in the United States, 2007-2010. HIV Surveillance Supplemental Report 2012.

Djan, D. (2018). "factors affecting the attitude of young people towards HIV testing uptake in rural Ghana”. j HIV retrovirus vol. 4 No. 2:13 doi: 10.21767/9676.100045

Hayes, R., et al. (2017). "A universal testing and treatment intervention to improve HIV control: one-year results from intervention communities in Zambia in the HPTN 071 (PopART) cluster-randomised trial." 14(5): e1002292.

Gumede, S. D., \& Sibiya, M. N. (2018). Health care users' knowledge, attitudes and perceptions of HIV self-testing at selected gateway clinics at eThekwini district, KwaZulu-Natal province, South Africa. SAHARA J : journal of Social Aspects of HIV/AIDS Research Alliance, 15(1), 103-109. doi:10.1080/1 7290376.2018.1517607

HIV/AIDS, J. U. N. P. o. and W. H. O. J. G. G. Scholar "UNAIDS/
WHO policy statement on HIV testing. 2004."

Joint United Nations Programme on HIV/AIDS and the World Health Organization, 2009.

Kippax S. A public health dilemma: A testing question. AIDS Care. 2006;18(3):230-5. [PubMed]

Logan B \& Laurel C, (2017), The routine offer of HIV testing in primary care settings: A review of the evidence, Canada.

Manongi, R., et al. (2014). "Knowledge, attitudes and acceptability to provider-initiated HIV testing and counseling: patients' perspectives in Moshi and Rombo Districts, Tanzania", Kilimanjaro Christian Medical Centre, Box 3010,Moshi,Kilimanjaro, Tanzania.

Ministry of Health (2017) Zambia, Lusaka.

Ministry of Health: Zambia country report, 2015

Nally A. et all (2019). Factors Affecting HIV Testing among Youth in Kenya. St. Petersburg, FL 33701 publishers. USA.

Nothling, Jani \& Kagee, Ashraf. (2013). Acceptability of routine HIV counselling and testing among a sample of South African students: Testing the Health Belief Model. African Journal of AIDS Research. 12. 141-150. 10.2989/16085906.2013.863214 Natasha S. C, (2016), Experiences Implementing a Routine HIV Screening Program in Two Federally Qualified Health Centers in the Southern United States.

Peralta L, Deeds BG, Young K. Promoting identification of HIV infected youths; borrowing concepts from the media to reduce the HIV epidemic. America

PEPFAR (2017) 'PEPFAR Zambia Country Operational Plan (COP) 2017 Strategic Direction Summary' [pdf]

Powers M, Faden R. (2006) Social Justice: The moral foundations of public health and health policy. Oxford: Oxford University Press.

Qiao. S., Zhang. L and Menon. J. (2018), Facilitators and barriers to HIV testing in Zambia: a systematic review of multilevel factors. PloS ONE 13(2): e0192327

UNAIDS. 90-90-90: An ambitious treatment target to help end the AIDS epidemic. In: HIV/AIDS JUNPo, editor. Geneva 2014. UNAIDS: Global education and information on HIV/AIDS, 2018

UNICEF HIV/AIDS report, Prevention among young people 2011

UNICEF, an assessment of HIV/AIDS youths participation (2011

UNAIDS report on AIDS 2010

Wanyenze, R. K., et al. (2008). "Acceptability of routine HIV counselling and testing, and HIV seroprevalence in Ugandan hospitals." 86: 302-309.

WHO: The Right to know, New Approaches to HIV testing and counselling. 2017.

World Health Organization. HIV testing and counselling: the gateway to treatment, care and support. Geneva: WHO; 2003.

World Health Organization: Zambia HIV country profile, 2019. Available at http://cfs.hivci.org/country-factsheet.htm,

Youssef, E. et al (2018), Factors associated with testing for $\mathrm{HIV}$ in people aged $\geq 50$ years: aqualitative stude, BMC public health. 